\title{
Antiresorptive and anabolic medications used in the perioperative period of patients with osteoporosis undergoing spine surgery: their impact on the biology of fusion and systematic review of the literature
}

\author{
Jean-Paul Bryant, MSc, Roberto J. Perez-Roman, MD, S. Shelby Burks, MD, and \\ Michael Y. Wang, MD \\ Department of Neurological Surgery, University of Miami Miller School of Medicine, Miami, Florida
}

OBJECTIVE Osteoporosis represents the most common metabolic disease of the bone, with an estimated $10 \%$ of adults aged 50 years or older affected in the United States. This patient population is at increased risk for spine fracture and instrumentation-related complications after spine surgery. Surgeon knowledge of the available treatments for patients with low bone mineral density (BMD) and how they impact biology of fusion may help mitigate negative effects in the postoperative period. Recombinant parathyroid hormone, which is sold under the name teriparatide, is the most extensively studied bone-protecting agent in humans. Additionally, the success of the monoclonal antibody denosumab has led to further clinical investigations of human patients undergoing spine surgery. Another monoclonal antibody, romosozumab, was recently approved by the US FDA for human use in patients with osteoporosis. Although studies of romosozumab in patients undergoing spine surgery have not been conducted, this is a promising potential therapeutic agent based on its early success in preclinical and clinical trials. Here, the authors aimed to review the mechanisms of action and evidence of use of antiresorptive and anabolic agents in patients with osteoporosis undergoing spine surgery.

METHODS In accordance with the PRISMA (Preferred Reporting Items for Systematic Reviews and Meta-Analyses) guidelines, a systematic review was conducted to explore the antiresorptive and anabolic agents used in the perioperative period in patients with osteoporosis undergoing spinal surgery. The search was performed by using the PubMed, Embase, and Cochrane Library databases. Titles and abstracts were screened and subsequently selected for full review. RESULTS The initial search returned 330 articles. Of these articles, 23 final articles were included and reviewed. Many of these articles reported that use of adjuvant agents in the perioperative period improved radiographic evidence of bony fusion and bone fusion rates. These agents tended to improve BMD postoperatively.

CONCLUSIONS Although antiosteoporosis agents are effective to varying degrees as treatments of patients with low $B M D$, teriparatide and bisphosphonates have been the most extensively studied with respect to spinal instrumentation. The advent of newer agents represents an area for further exploration, especially due to the current paucity of controlled investigations. It is imperative for spine surgeons to understand the mechanisms of action of these drugs and their effects on biology of fusion.

https://thejns.org/doi/abs/10.3171/2021.3.FOCUS201049

KEYWORDS osteoporosis; spine surgery; fusion; antiresorptive; anabolic

$\mathrm{O}$ STEOPOROSIS is the most common metabolic bone disease, with an estimated $10.3 \%$ of adults aged 50 years or older affected in the United States. ${ }^{1,2}$ As the US population continues to age, the incidence of disease and complications due to osteoporosis has steadily increased. Although osteoporosis is more common in women, $4 \%-6 \%$ of men older than 50 years also have osteoporosis, with another 33\%-47\% developing osteopenia. ${ }^{3-6}$ The predisposition to fracture in people with osteoporosis has been well delineated, with 1 in 2 women older than 50 years and 1 in 3 men older than 70 years affected by low-trauma fractures.? Reports have cited as many as

ABBREVIATIONS ATP = adenosine triphosphate; BMD = bone mineral density; ODI = Oswestry Disability Index; OPG = osteoprotegerin; PLIF = posterior lumbar interbody fusion; PRISMA = Preferred Reporting Items for Systematic Reviews and Meta-Analyses; PTH = parathyroid hormone; RANK = receptor of RANKL; RANKL = receptor activator of nuclear factor kappa B ligand.

SUBMITTED December 7, 2020. ACCEPTED March 24, 2021.

INCLUDE WHEN CITING DOI: 10.3171/2021.3.FOCUS201049. 


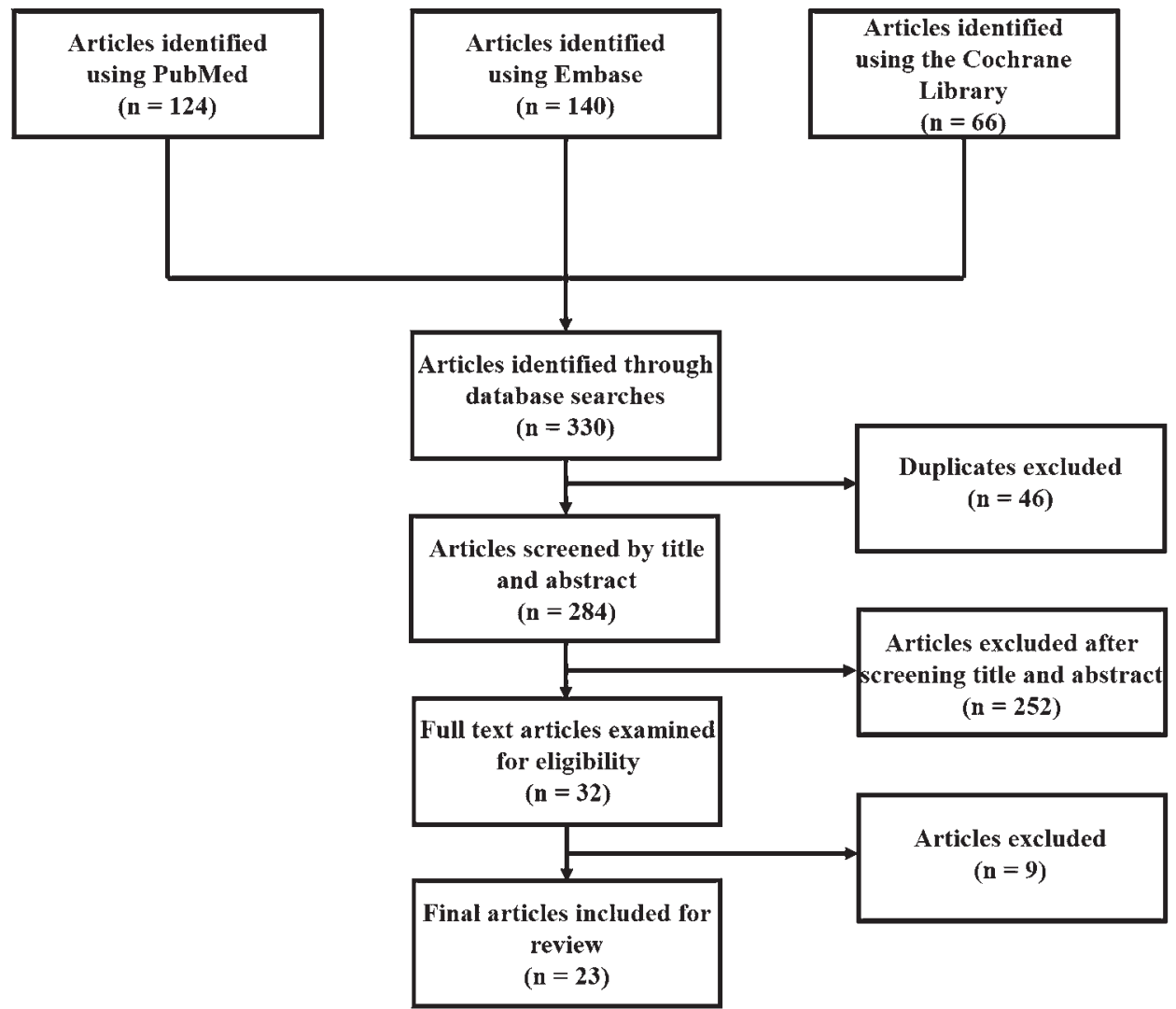

FIG. 1. PRISMA flowchart showing the search and review process used to select articles for this systematic review.

700,000 osteoporotic spine fractures per year, with postmenopausal white females at the greatest lifetime risk for vertebral fracture. ${ }^{8}$

Those afflicted with osteoporosis present with unique challenges when undergoing spinal surgery. Instrumentation failure is a commonly cited complication of patients with osteoporosis who have undergone surgical intervention, with studies showing that decreased bone mineral density (BMD) reduces the effectiveness of pedicle fixation strength, increases the incidence of cage subsidence, and increases the risk of iatrogenic fracture and proximal junctional kyphosis. ${ }^{9-15}$ Placement of spinal column instrumentation in someone with low or decreased BMD often mandates the use of special strategies, including iliac fixation techniques, extension of fixation points, and cement augmentation, as well as acceptance of a more modest correction. ${ }^{8}$ Despite these technical strategies, the risk of complications from spine surgery remains high in patients with poor bone stock. This has elicited a need for improved medical therapy in the perioperative period. Numerous drugs have been shown to be efficacious for reducing mechanical complications, and patients have demonstrated improvement in bone microarchitecture. ${ }^{16-21}$ These medications are frequently prescribed in the clinical setting and have been well described; however, few comprehensive reviews that also include recently discovered therapeutic agents exist in the spine literature. In this paper, we present an updated systematic review of the antiresorptive and anabolic agents used in the perioperative period in patients with osteoporosis undergoing spinal fusion.

\section{Methods}

A systematic review was conducted according to the Preferred Reporting Items for Systematic Reviews and Meta-Analyses (PRISMA) guidelines to investigate the use of antiresorptive and anabolic agents in spine surgery patients with osteoporosis (Fig. 1). We used the PubMed MEDLINE (National Library of Medicine), Embase (Elsevier), and Cochrane Library (Wiley) databases to conduct the search. The search terms used for each database are listed in Table 1.

Duplicates were excluded after completion of the search. The titles and abstracts of the remaining articles were screened. These remaining articles were subsequently screened for final review on the basis of the following inclusion criteria: the study was a peer-reviewed and full-length article; the full-length text was available in English; the study included patients with osteoporosis (or, in the setting of a preclinical investigation, involved an osteoporotic animal model); the study patients underwent a spinal surgical procedure; the study was not a review article; and the study patients received bisphosphonates, romosozumab, denosumab, or teriparatide. After final exclusion, the remaining articles were reviewed to determine the antiresorptive or anabolic agent administered, number 
TABLE 1. Search terms entered into the PubMed, Embase, and Cochrane Library databases and number of results returned

\begin{tabular}{lccc}
\hline \multicolumn{1}{c}{ Database } & Search Terms & $\begin{array}{c}\text { No. of Results } \\
\text { Returned }\end{array}$ \\
\hline $\begin{array}{l}\text { PubMed MEDLINE (Na- } \\
\text { tional Library of Medicine) }\end{array}$ & $\begin{array}{c}\text { ((osteoporosis OR osteoporotic) AND (agent OR (drug)) AND (surgery OR ("spine surgery")) AND } \\
\text { (spinal[TITLE] OR fusion[TITLE])) OR ((rabbit OR rat) AND fusion[TITLE] AND "parathyroid hormone") }\end{array}$ \\
\hline Embase (Elsevier) & $\begin{array}{c}\text { (osteoporosis OR osteoporotic) AND (agent OR drugs) AND ((surgery OR "spine surgery") AND (spinal:ti } \\
\text { OR fusion:ti) OR ((rabbit OR rat) AND fusion:ti AND "parathyroid hormone")) }\end{array}$ & 140 \\
\hline Cochrane Library (Wiley) & $\begin{array}{c}\text { ((osteoporosis OR osteoporotic) AND (agent OR (drug)) AND (surgery OR ("spine surgery")) AND } \\
\text { (spinal[TITLE] OR fusion[TITLE])) OR ((rabbit OR rat) AND fusion[TITLE] AND "parathyroid hormone") }\end{array}$ \\
\hline
\end{tabular}

of patients (or animals and species if a preclinical investigation), surgical procedure performed, and study outcome (Tables 2 and 3).

\section{Results}

The initial search returned 330 articles (Fig. 1). Of these articles, 23 final articles were included and reviewed. Many of the final articles found that the use of adjuvant agents in the perioperative period improved radiographic evidence of bony fusion. Here, we review these studies on the basis of the antiresorptive or anabolic properties of the agents used. An exhaustive summary of all studies included is displayed in Tables 2 and 3.

\section{Antiresorptive Agents Bisphosphonates}

It has been well over a decade since their advent, and bisphosphonates remain the first-line treatment of osteoporosis. Preclinical and clinical studies have demonstrated that bisphosphonates have therapeutic potential for spine surgery patients with osteoporosis. ${ }^{22-24}$ The first randomized controlled trial to examine the effects of bisphosphonates in the perioperative period after spinal fusion was conducted by Nagahama et al. in 2011 (Table 2). ${ }^{25}$ In their study, 40 patients underwent single-level posterior lumbar interbody fusion (PLIF) and were randomly assigned to an alendronate or control group (that received vitamin D supplementation). At 1 year postoperatively, the alendronate group had a significantly higher fusion rate $(p=0.025)$, significantly decreased incidence of vertebral compression fracture $(\mathrm{p}=0.027)$, and decreased rate of cage subsidence (5\% in the alendronate group vs $29 \%$ in the control group), although this difference was not statistically significant. On clinical assessment, Oswestry Disability Index (ODI) scores did not differ significantly between groups; however, mean ODI scores consistently decreased until 1 year after surgery in the alendronate group, whereas mean ODI score plateaued at the 3-month follow-up in the control group.

A retrospective analysis by Park et al. delineated the effects of zoledronic acid on mean volume of fusion mass in patients with symptomatic degenerative lumbar spinal stenosis who underwent one- or two-level posterolateral fusion. ${ }^{26}$ Patients were divided into four groups according to treatment: autograft and zoledronic acid; allograft and zoledronic acid; autograft alone; and allograft alone.
Additionally, clinical outcomes were assessed with scores on the visual analog scale, ODI, and 36-Item Short-Form Health Survey. The mean fusion mass per level and clinical outcomes at the 6-month follow-up were similar between groups. This study suggests that although zoledronic acid does not decrease the volume of fusion mass, it appears to have no clinically or statistically significant benefit compared with graft alone.

Tu et al. subsequently conducted a retrospective analysis of 64 patients with degenerative spondylolisthesis and osteoporosis who underwent lumbar interbody fusion. ${ }^{27}$ Half the patients $(n=32)$ were treated with intravenous infusion of zoledronic acid, and the remaining half received no treatment. At the final follow-up (2 years), the zoledronic acid group had a significantly decreased incidence of vertebral compression fracture $(p=0.006)$, pedicle screw loosening $(p=0.03)$, and cage subsidence $(p=0.04)$ when compared with the control group. These results were bolstered in a randomized controlled trial conducted by Chen et al. that examined the effects of zoledronic acid on bone fusion in 79 patients with osteoporosis who underwent single-level PLIF. ${ }^{28}$ Patients were randomly assigned to receive either zoledronic acid or saline infusion. Bridging bone grade $\mathrm{A}$ or $\mathrm{B}$-defined as bridging bone bonding with adjacent or superior/inferior vertebral bodieswas observed more frequently at the 3-, 6-, and 9-month follow-ups $(p<0.05)$ in the treatment group. The group treated with zoledronic acid had 0 patients who experienced vertebral compression fracture compared with 6 in the control group $(\mathrm{p}<0.05)$. Finally, Seki et al. conducted a prospective study of 58 women with osteoporosis who underwent correction for adult spinal deformity. ${ }^{29}$ These patients received either teriparatide or low-dose bisphosphonates. Outcomes were assessed with preoperative and postoperative CT and radiographic images. Additionally, pain scores and ODI scores were recorded preoperatively and 2 years postoperatively. The fusion rate was significantly higher in the teriparatide group (89\%) than in the bisphosphonate group $(77 \%)(\mathrm{p}=0.0002)$. Preoperative and postoperative clinical scores significantly improved after surgery in both groups.

Given the significant results of these studies, it is evident that bisphosphonates can help ameliorate the deleterious effects of spinal surgery in patients with osteoporosis. ${ }^{30-33}$ However, the most recent study that compared bisphosphonates with teriparatide suggests that the latter may be a more effective agent to use in the perioperative 
Bryant et al.

TABLE 2. Summary of human studies on use of anabolic or antiresorptive agents in patients who underwent spinal surgery

\begin{tabular}{|c|c|c|c|c|}
\hline $\begin{array}{c}\text { Authors \& } \\
\text { Year }\end{array}$ & $\begin{array}{l}\text { Agent } \\
\text { Used }\end{array}$ & $\begin{array}{c}\text { No. of } \\
\text { Patients }\end{array}$ & $\begin{array}{l}\text { Surgical } \\
\text { Procedure }\end{array}$ & Outcome \\
\hline $\begin{array}{l}\text { Nagahama } \\
\text { et al., } \\
2011^{25}\end{array}$ & Alendronate & 40 & PLIF & $\begin{array}{l}\text { Treatment group had significantly greater fusion rate }(p=0.025) \& \text { decreased incidence } \\
\text { of vertebral compression fracture }(p=0.027) \text {. Decreased risk of cage subsidence } \\
\text { was observed, but difference was not significant }(5 \% \text { in alendronate group vs } 29 \% \text { in } \\
\text { control group). }\end{array}$ \\
\hline $\begin{array}{l}\text { Park et al., } \\
2013^{26}\end{array}$ & $\begin{array}{l}\text { Zoledronic } \\
\quad \text { acid }\end{array}$ & 44 & $\begin{array}{l}\text { 1- or 2-level pos- } \\
\text { terolat fusion }\end{array}$ & $\begin{array}{l}\text { No significant differences in mean fusion mass per level btwn all groups \& no significant } \\
\text { differences in clinical outcome based on VAS, ODI, \& SF-36 scores. }\end{array}$ \\
\hline $\begin{array}{l}\text { Tu et al., } \\
2014^{27}\end{array}$ & $\begin{array}{l}\text { Zoledronic } \\
\text { acid }\end{array}$ & 64 & $\begin{array}{l}\text { Lumbar interbody } \\
\text { fusion }\end{array}$ & $\begin{array}{l}\text { Zoledronic acid group had significantly decreased incidence of vertebral compression } \\
\text { fracture }(p=0.006) \text {, pedicle screw loosening }(p=0.03) \text {, \& cage subsidence }(p=0.04) \\
\text { compared w/ control group. }\end{array}$ \\
\hline $\begin{array}{l}\text { Chen et } \\
\text { al., } 2016^{28}\end{array}$ & $\begin{array}{l}\text { Zoledronic } \\
\text { acid }\end{array}$ & 79 & Single-level PLIF & $\begin{array}{l}69 \text { patients completed } 1 \text {-yr FU. Bridging bone was more frequently observed at 3-, } 6-\text {, } \\
\& 9 \text {-mo FUs }(p<0.05) \text { in zoledronic acid group than control group. } 0 \text { patients in zole- } \\
\text { dronic acid group had vertebral compression fracture vs } 6 \text { in control group }(p<0.05) \text {. }\end{array}$ \\
\hline $\begin{array}{l}\text { Seki et al., } \\
2017^{29}\end{array}$ & $\begin{array}{l}\text { Alendronate } \\
\text { or risedronate } \\
\text { vs teriparatide }\end{array}$ & 58 & $\begin{array}{l}\text { Multilevel posterolat } \\
\text { fusion for spinal } \\
\text { deformity correction }\end{array}$ & $\begin{array}{l}\text { Fusion rate was significantly greater in teriparatide group ( } 89 \%) \text { than bisphosphonate } \\
\text { group }(77 \%)(p=0.0002) \text {. Pain scores \& ODI scores were significantly improved in } \\
\text { both groups postop. }\end{array}$ \\
\hline $\begin{array}{l}\text { Ding et al., } \\
2017^{32}\end{array}$ & $\begin{array}{l}\text { Zoledronic } \\
\text { acid }\end{array}$ & 94 & TLIF & $\begin{array}{l}64 \text { patients who completed final FU were retrospectively examined. } 30 \text { patients were } \\
\text { given zoledronic acid from } 3 \text { to } 5 \text { days postop. Spinal fusion rate was significantly } \\
\text { greater }(p<0.05) \text { in zoledronic acid group than control group at } 12 \text { mos. Adjacent } \\
\text { vertebral compression fracture occurred in } 5 \text { control patients vs } 0 \text { patients treated w/ } \\
\text { zoledronic acid. }\end{array}$ \\
\hline $\begin{array}{l}\text { Kang et } \\
\text { al., } 2019^{33}\end{array}$ & Alendronate & 97 & Single-level PLIF & $\begin{array}{l}63 \text { postmenopausal women eligible for single-level PLIF who were long-term alendro- } \\
\text { nate users were compared w/ } 34 \text { patients who were nonusers. Serum CTX levels } \\
\text { dramatically decreased in alendronate group }(p<0.05) \text {. There was no significant } \\
\text { difference in ODI or VAS scores. }\end{array}$ \\
\hline $\begin{array}{l}\text { Kawa- } \\
\text { bata et al., } \\
2020^{30}\end{array}$ & $\begin{array}{l}\text { Bisphos- } \\
\text { phonates vs } \\
\text { teriparatide }\end{array}$ & 159 & $\begin{array}{l}\text { Posterior instru- } \\
\text { mented fusion }\end{array}$ & $\begin{array}{l}\text { Patients underwent posterior instrumented fusion for osteoporotic vertebral fracture. } \\
\text { Mechanical complication rate was significantly decreased in teriparatide group ( } p= \\
\text { 0.045). Screw backout rate was also significantly decreased in teriparatide group ( } p= \\
\text { 0.024). }\end{array}$ \\
\hline $\begin{array}{l}\text { Ohtori et } \\
\text { al., } 2012^{34}\end{array}$ & Teriparatide & 57 & $\begin{array}{l}\text { Posterolat lumbar } \\
\text { fusion }\end{array}$ & $\begin{array}{l}79 \% \text { of teriparatide group had successful fusion vs } 65 \% \text { of bisphosphonate group. Aver- } \\
\text { age duration of bone union was } 8 \text { mos in teriparatide group vs } 10 \text { mos in bisphospho- } \\
\text { nate group. Both comparisons were significant. }\end{array}$ \\
\hline $\begin{array}{l}\text { Ebata et } \\
\text { al., } 2017^{37}\end{array}$ & Teriparatide & 66 & TLIF or PLIF & $\begin{array}{l}\text { At } 4 \& 6 \text { mos after PLIF or TLIF, no. of patients w/ bone fusion was significantly greater } \\
\text { in teriparatide group than control arm. At } 6 \text {-mo FU, } 69 \% \text { of the teriparatide group } \\
\text { showed complete fusion vs } 35.1 \% \text { of control arm }(p=0.013) \text {. }\end{array}$ \\
\hline $\begin{array}{l}\text { Inoue et } \\
\text { al., } 2014^{18}\end{array}$ & Teriparatide & 29 & $\begin{array}{l}\text { Thoracic \&/or lum- } \\
\text { bar spinal fusion }\end{array}$ & $\begin{array}{l}\text { Mean insertional torque of pedicle screws was significantly greater in teriparatide group } \\
(1.28 \pm 0.42 \mathrm{Nm}) \text { than control group }(1.08 \pm 0.52 \mathrm{Nm})(\mathrm{p}<0.01) \text {. Average no. of levels } \\
\text { fused was significantly greater in teriparatide group }(4.2 \pm 2.0) \text { than control group }(2.3 \\
\pm 1.7) \text {. }\end{array}$ \\
\hline $\begin{array}{l}\text { Cho et al., } \\
2017^{38}\end{array}$ & Teriparatide & 47 & $\begin{array}{l}\text { PLIF w/ pedicle } \\
\text { screw fixation }\end{array}$ & $\begin{array}{l}\text { Average time to interbody fusion was significantly shorter in teriparatide group }(6.0 \pm 4.8 \\
\text { mos) than bisphosphonate group }(10.4 \pm 7.2 \mathrm{mos})(p=0.006) \text { at } 24 \text {-mo FU. }\end{array}$ \\
\hline $\begin{array}{l}\text { Kim et al., } \\
2018^{39}\end{array}$ & Teriparatide & 84 & $\begin{array}{l}\text { TLIF \& pedicle } \\
\text { screw fixation }\end{array}$ & $\begin{array}{l}\text { At } 6-12 \text { mos postop, teriparatide group }(2.3 \%) \text { showed significantly fewer loose pedicle } \\
\text { screws than bisphosphonate group }(9.2 \%)(p<0.05) \text {. Teriparatide group }(14.86 \% \pm \\
14.97 \%) \text { showed significantly greater improvement in T-score than bisphosphonate } \\
\text { group }(8.55 \% \pm 11.43 \%)(p<0.05) \text {. }\end{array}$ \\
\hline $\begin{array}{l}\text { Ide et al., } \\
2018^{65}\end{array}$ & $\begin{array}{l}\text { Teriparatide \& } \\
\text { denosumab }\end{array}$ & 16 & PLIF & $\begin{array}{l}\text { Radiographic CT revealed significantly greater rates of bone union }(82 \% \text { vs } 36 \%, p< \\
0.05) \& \text { spinal fusion }(p<0.05) \text { in combination treatment group than teriparatide group } \\
\text { at } 6 \text { mos. }\end{array}$ \\
\hline $\begin{array}{l}\text { Oba et al., } \\
2020^{31}\end{array}$ & $\begin{array}{l}\text { Teriparatide \& } \\
\text { bisphospho- } \\
\text { nates }\end{array}$ & 104 & $\begin{array}{l}\text { Multilevel interbody } \\
\quad \text { fusion }\end{array}$ & $\begin{array}{l}\text { Radiological evaluation \& bone fusion evaluation were performed w/ CT. Bone fusion rate } \\
\text { at } 6 \text { mos postop tended to be greater in bisphosphonate group. There were no signifi- } \\
\text { cant differences in bone fusion score btwn teriparatide \& bisphosphonate groups. }\end{array}$ \\
\hline
\end{tabular}

CTX = C-terminal cross-linking telopeptide; FU = follow-up; SF-36 = 36-Item Short-Form Health Survey; TLIF = transforaminal lumbar interbody fusion; VAS = visual analog scale. 
TABLE 3. Summary of preclinical animal studies on use of teriparatide in animals that underwent spinal surgery

\begin{tabular}{|c|c|c|c|c|c|}
\hline $\begin{array}{l}\text { Authors \& } \\
\text { Year }\end{array}$ & $\begin{array}{l}\text { Agent } \\
\text { Used }\end{array}$ & $\begin{array}{c}\text { No. of } \\
\text { Animals }\end{array}$ & Model & $\begin{array}{l}\text { Surgical } \\
\text { Procedure }\end{array}$ & Outcome \\
\hline $\begin{array}{l}\text { Lawrence } \\
\text { et al., } \\
2006^{41}\end{array}$ & Teriparatide & 56 & $\begin{array}{l}\text { Ovariecto- } \\
\text { mized rat }\end{array}$ & $\begin{array}{l}\text { Posterolat lumbar } \\
\text { fusion }\end{array}$ & $\begin{array}{l}\text { Teriparatide group had a fusion rate of } 52 \% \text { vs } 37 \% \text { in control group. } \\
\text { Greater osteocalcin level was reported in the experimental group ( } 88.6 \\
\mathrm{ng} / \mathrm{L}) \text { than control group }(59.8 \mathrm{ng} / \mathrm{L}) \text {. }\end{array}$ \\
\hline $\begin{array}{l}\text { Qiu et al., } \\
2013^{42}\end{array}$ & Teriparatide & 36 & $\begin{array}{l}\text { Ovariecto- } \\
\text { mized rat }\end{array}$ & $\begin{array}{l}\text { Bilat posterolat } \\
\text { intertransverse } \\
\text { process fusion }\end{array}$ & $\begin{array}{l}\text { Average radiographic fusion scores were significantly greater at } 4 \text { wks } \\
(2.03 \text { vs } 1.45, p<0.05) \& 6 \text { wks }(3.66 \text { vs } 2.56, p<0.01) \text { in PTH group } \\
\text { than control group. Osteocalcin levels were significantly greater at } 4 \text { wks } \\
(81.42 \pm 12.46 \text { vs } 41.67 \pm 9.42) \& 6 \text { wks }(71.21 \pm 11.38 \text { vs } 39.83 \pm 6.52) \\
(p<0.05) \text { in the experimental group that received recombinant PTH } \\
\text { than control group. Type I collagen levels were significantly greater at } 4 \\
\text { wks in PTH group }(84.47 \pm 11.93) \text { than control group }(68.39 \pm 8.27) \text { ( } p \\
<0.05) \text {. }\end{array}$ \\
\hline $\begin{array}{l}\text { O'Loughlin } \\
\text { et al., } \\
2009^{43}\end{array}$ & Teriparatide & 44 & Rabbit & $\begin{array}{l}\text { Bilat posterolateral } \\
\text { spine fusion }\end{array}$ & $\begin{array}{l}\text { Animals were euthanized \& assessed at } 6 \text { wks. PTH group exhibited } \\
\text { significantly greater fusion }(p<0.01) \text {, greater fusion mass volume }(p \\
<0.001) \text {, increased osteoblast quantity }(p<0.001) \text {, \& higher median } \\
\text { percentage bone area }(p<0.001) \text { on histological evaluation. }\end{array}$ \\
\hline $\begin{array}{l}\text { Lehman et } \\
\text { al., } 2010^{44}\end{array}$ & Teriparatide & 51 & Rabbit & $\begin{array}{l}\text { Intertransverse } \\
\text { process fusion }\end{array}$ & $\begin{array}{l}\text { Comparison of PTH w/ calcitonin administration showed that teriparatide } \\
\text { was superior to calcitonin on histologic \& radiographic evaluation. Aver- } \\
\text { age Emery grade score was significantly greater in teriparatide group } \\
(6.26 \pm 0.93) \text { than calcitonin cohort }(5.99 \pm 1.46)(p<0.031) \text {. }\end{array}$ \\
\hline $\begin{array}{l}\text { Sugiura et } \\
\text { al., } 2015^{40}\end{array}$ & Teriparatide & 18 & $\begin{array}{l}\text { Glucocorti- } \\
\text { coid-induced } \\
\text { osteoporotic } \\
\text { rat }\end{array}$ & Posterolat fusion & $\begin{array}{l}\text { 8-wk-old male rats were given methylprednisolone for } 12 \text { wks. After } 6 \text { wks, } \\
\text { rats underwent } L 4-5 \text { spinal fusion. Rats were given teriparatide or saline } \\
5 \text { times/wk for } 6 \text { wks. Bone volume \& bone microstructural parameters } \\
\text { were significantly greater in teriparatide group than control group ( } p< \\
\text { 0.05). Fusion rate was higher in teriparatide group }(89 \%) \text { than control } \\
\text { group }(56 \%) \text {. }\end{array}$ \\
\hline $\begin{array}{l}\text { Nakao et } \\
\text { al., } 2011^{23}\end{array}$ & Alendronate & 46 & $\begin{array}{l}\text { Ovariecto- } \\
\text { mized rat }\end{array}$ & $\begin{array}{l}\text { Posterolat inter- } \\
\text { transverse process } \\
\text { fusion }\end{array}$ & $\begin{array}{l}8 \text { wks after sham procedure or bilat ovariectomy, animals underwent L4-5 } \\
\text { fusion. Animals received saline or alendronate } 1 \text { time/wk for } 8 \text { wks. } \\
\text { Cross-sectional area of fusion mass was larger in alendronate group } \\
\text { than control group }(p<0.05) \text {. Histology revealed greater new bone } \\
\text { formation in alendronate-treated animals. }\end{array}$ \\
\hline $\begin{array}{l}\text { Yasen et } \\
\text { al., } 2015^{22}\end{array}$ & $\begin{array}{l}\text { Zoledronic } \\
\text { acid }\end{array}$ & 50 & $\begin{array}{l}\text { Ovariecto- } \\
\text { mized rat }\end{array}$ & Bilat lumbar fusion & $\begin{array}{l}8 \text { wks after ovariectomy, animals underwent spinal fusion w/ zoledronic } \\
\text { acid or saline. Lumbar spines were harvested \& processed. Manual } \\
\text { palpation test \& radiographic scales differed significantly btwn high-dose } \\
\text { zoledronic acid \& control groups, w/ zoledronic acid group exhibiting } \\
\text { improved outcomes }(p<0.05) \text {. Bone volume fraction was significantly } \\
\text { greater in experimental group }(p<0.01) \text {. }\end{array}$ \\
\hline $\begin{array}{l}\text { Yishake et } \\
\text { al., } 2018^{24}\end{array}$ & $\begin{array}{l}\text { Zoledronic } \\
\text { acid w/ or } \\
\text { w/o teripa- } \\
\text { ratide }\end{array}$ & 60 & $\begin{array}{l}\text { Ovariecto- } \\
\text { mized rat }\end{array}$ & $\begin{array}{l}\text { Bilat posterolat } \\
\text { intertransverse } \\
\text { process fusion }\end{array}$ & $\begin{array}{l}\text { Rats were ovariectomized \& underwent L4-5 intertransverse fusion after } \\
10 \text { mos. Teriparatide \& zoledronic acid combination therapy increased } \\
\text { lumbar bone volume \& fusion rate. Combination therapy also increased } \\
\text { bone formation rate. }\end{array}$ \\
\hline
\end{tabular}

period. In addition to the aforementioned studies, Ohtori et al. conducted multiple trials that examined bisphosphonate use after spinal surgery. ${ }^{17,19,34}$ Because these studies mainly focused on the efficacy of teriparatide, they are included in the next section (Teriparatide).

There are many well-documented adverse effects of bisphosphonates, which can be categorized as skeletal and extraskeletal. Skeletal effects include osteonecrosis of the jaw and atypical femur fracture. Extraskeletal effects include gastrointestinal adverse effects such as esophagitis, upper gastrointestinal bleeding, and esophageal ulcer. Additionally, patients have reported new-onset atrial fibrillation through a mechanism that is unclear (Table 4). ${ }^{35}$

\section{Anabolic Agents Teriparatide}

Recombinant parathyroid hormone (PTH), known as teriparatide, is one of three anabolic agents currently approved for the treatment of osteoporosis in the United States. In the clinical setting, daily administration of subcutaneous teriparatide has been associated with improved BMD and decreased fracture risk in postmenopausal women with osteoporosis. In a meta-analysis of 8 randomized controlled trials, 6 reported fracture risk and found that teriparatide treatment was associated with reduced risk of vertebral fracture $(70 \%$ risk reduction, risk ratio 
TABLE 4. Dosage and route of administration of the agents reviewed in the present study

\begin{tabular}{|c|c|c|c|c|}
\hline $\begin{array}{c}\text { Agent (medication } \\
\text { name) }\end{array}$ & $\begin{array}{l}\text { Standard } \\
\text { Dosage }\end{array}$ & $\begin{array}{c}\text { Route of } \\
\text { Administration }\end{array}$ & $\begin{array}{l}\text { Duration of } \\
\text { Treatment }\end{array}$ & Contraindications \\
\hline $\begin{array}{l}\text { Bisphosphonates } \\
\text { (alendronate) }\end{array}$ & $\begin{array}{l}\text { For patients at high risk of frac- } \\
\text { ture, } 70 \mathrm{mg} \text { once weekly or } \\
10 \mathrm{mg} \text { once daily; for those } \\
\text { not at high risk, } 35 \mathrm{mg} \text { once } \\
\text { weekly or } 5 \mathrm{mg} \text { once daily }\end{array}$ & Oral & $\begin{array}{l}\text { Optimal duration not estab- } \\
\text { lished, but possibly as } \\
\text { long as } 10 \text { yrs if fracture } \\
\text { risk remains high }\end{array}$ & $\begin{array}{l}\text { Hypersensitivity to bisphosphonates or their } \\
\text { components (if susceptible), hypocalcemia, } \\
\text { esophageal abnormalities (which can delay } \\
\text { emptying), increased risk of aspiration, inabil- } \\
\text { ity to remain upright for at least } 30 \text { mins }\end{array}$ \\
\hline $\begin{array}{l}\text { Teriparatide } \\
\text { (Forteo) }\end{array}$ & $20 \mu \mathrm{g} / \mathrm{day}$ & $\begin{array}{l}\text { Subcutaneous } \\
\text { injection }\end{array}$ & Up to $2 \mathrm{yrs}$ & $\begin{array}{l}\text { Hypersensitivity to teriparatide or components, if } \\
\text { susceptible (e.g., angioedema or anaphylaxis) }\end{array}$ \\
\hline $\begin{array}{l}\text { Denosumab } \\
\text { (Prolia) }\end{array}$ & 60 mg every 6 mos & $\begin{array}{l}\text { Subcutaneous } \\
\text { injection }\end{array}$ & $\begin{array}{l}\text { BMD maintenance w/ contin- } \\
\text { ued use as long as } 10 \mathrm{yrs}\end{array}$ & Hypocalcemia \& pregnancy \\
\hline $\begin{array}{l}\text { Romosozumab } \\
\text { (Evenity) }\end{array}$ & 210 mg once monthly & $\begin{array}{l}\text { Subcutaneous } \\
\text { injection }\end{array}$ & $12 \mathrm{mos}$ & $\begin{array}{l}\text { Hypersensitivity to romosozumab or its com- } \\
\text { ponents, if susceptible (e.g., angioedema or } \\
\text { anaphylaxis), \& hypocalcemia }\end{array}$ \\
\hline
\end{tabular}

$0.30,95 \%$ CI $0.21-0.44 ; 3$ trials) and nonvertebral fracture (38\% risk reduction, risk ratio $0.62,95 \%$ CI $0.44-0.87$; 3 trials). ${ }^{36}$ On average, teriparatide increased spine BMD by $8.14 \%$ (95\% CI $6.72-9.55 \%$; 8 trials) and increased total hip BMD by $2.48 \%$ (95\% CI $1.67-3.29 \%$; 7 trials) in the trials that reported these outcomes.

Ohtori et al. conducted a prospective study to examine the effects of teriparatide on fusion rate in postmenopausal women with osteoporosis who underwent posterolateral lumbar fusion (Table 2). ${ }^{34}$ In total, 29 of 57 women enrolled were treated with teriparatide, and the remaining half were treated with bisphosphonates. In comparison with the cohort treated with bisphosphonates, the teriparatide-treated cohort had a significantly higher rate of fusion on CT evaluation ( $79 \%$ and $65 \%$, respectively; $p=0.02$ ) and a significantly shorter average time to bone union when evaluated with both CT $(p=0.03)$ and radiography $(p=0.04)$. This analysis was supported by a large, multicenter prospective study that explored osseous enhancement in patients with osteoporosis after lumbar interbody fusion. ${ }^{37}$ In this study, $49 \%$ of patients were randomly assigned to the teriparatide arm, and the remaining patients in the control arm did not receive teriparatide. Those patients who completed weekly subcutaneous teriparatide injection treatment (78\% of treatment arm) had a significantly greater rate of bone fusion 6 months postoperatively than the patients in the control $\operatorname{arm}(\mathrm{p}=0.013)$.

Another study by Inoue et al. alternatively described the effectiveness of teriparatide in the preoperative setting in postmenopausal women with osteoporosis (Table 2). ${ }^{18}$ Twenty-nine patients underwent thoracolumbar spinal fusion, and nearly half were treated with teriparatide preoperatively. The treatment cohort had significantly greater mean insertional torque of pedicle screws during surgery $(\mathrm{p}<0.01)$. Cho et al. saw similar results when examining the efficacy of teriparatide administration in women with osteoporosis undergoing PLIF (Table 2). ${ }^{38}$ The teriparatidetreated group showed a significantly higher fusion rate than the bisphosphonate group at 6 months after surgery $(77.8 \%$ vs $53.6 \%, p=0.006$ ). Furthermore, the teriparatide-treated group showed significantly higher BMD (represented by
T-score) at 2 years postoperatively than the bisphosphonate group ( 0.7 vs $0.1, p=0.013$ ). In addition to improved fusion rate and greater BMD, it has also been shown that teriparatide can affect pedicle screw loosening in patients with osteoporosis after a spinal procedure. ${ }^{39}$ In this study, 84 patients with osteoporosis who underwent transforaminal lumbar interbody fusion with pedicle screw fixation received either teriparatide or bisphosphates postoperatively for 6 months. In the 6 to 12 months after surgery, the teriparatide-treated group showed a significantly smaller number of patients with pedicle screw loosening than the bisphosphonate group (2.3\% vs $9.2 \%, \mathrm{p}<0.05)$. Moreover, the teriparatide group had a significantly higher T-score, representing greater BMD, than the bisphosphonate-treated group $(\mathrm{p}<0.05)$.

Studies of teriparatide have demonstrated increased spinal fusion rates after surgery in osteoporotic animal models (Table 3).$^{40}$ An early study by Lawrence et al. ${ }^{41}$ examined the effect of daily teriparatide injection on posterolateral lumbar fusion in a rat model. The PTH group had a fusion rate of 52\% compared with $37 \%$ in the control group. Since this study, ample evidence supporting these results has been reported by Lawrence and other researchers. Qiu et al. found that high-dose intermittent PTH administration augmented fusion quality and reduced healing time in ovariectomized osteoporotic female rats undergoing bilateral posterolateral intertransverse process fusion. ${ }^{42}$ The measured biomarkers followed this trend, in that levels of osteocalcin and type I collagen were significantly greater in the experimental group. Similarly, O'Loughlin et al. published encouraging results obtained in a rabbit model. ${ }^{43}$ The PTH-treated group exhibited significantly greater fusion, greater fusion mass volume, increased osteoblast quantity, and higher median bone area percentage on histological evaluation. When comparing PTH with calcitonin administration for intertransverse process fusion in a rabbit model, Lehman et al. found that teriparatide-treated animals showed superior results on histological and radiographic evaluations compared with calcitonin-treated animals, including greater Emery grade scores in the teriparatide-treated group. ${ }^{44}$ These results in 
both humans and animal models demonstrate that teriparatide is an efficacious agent for patients with osteoporosis undergoing spine surgery (Tables 2 and 3).

Discontinuation of teriparatide is recommended after 2 years of treatment owing to black box warnings for increased risk of osteosarcoma. However, these warnings were determined with long-term rodent trials, and only 1 case of osteosarcoma has been observed in humans. ${ }^{45}$ Additionally, teriparatide has been commonly associated with headache, nausea, dizziness, and muscle spasm in placebo-controlled trials. ${ }^{46}$

\section{Discussion}

We present an updated systematic review and discuss adjunctive medical therapy for patients with osteoporosis undergoing spinal procedures. Our search highlighted growing evidence about this topic, emphasizing the importance of understanding the therapeutic potential of antiresorptive and anabolic agents in the perioperative period. Here, we discuss the mechanisms of action of the currently available antiresorptive and anabolic agents, with particular focus on how these agents promote bone formation or prevent bone catabolism. Furthermore, we briefly summarize studies that have demonstrated the efficacy of monoclonal antibodies used to treat patients with osteoporosis.

\section{Mechanisms of Action of Bisphosphonates, Denosumab, Romosozumab, and Teriparatide}

Successful spinal fusion with new bone formation requires a mechanistically complex process. It is influenced by a multitude of factors, ranging from the methods of fixation to specific patient characteristics. ${ }^{47}$ At the cellular level, differentiation of mesenchymal and hematopoietic cell lines into osteoblasts and osteoclasts, respectively, is paramount. Osteoclast maturation requires the presence of several signaling molecules, including receptor activator of nuclear factor kappa B ligand (RANKL) and its receptor (RANK). Simultaneously, the presence of osteoprotegerin (OPG), a competitive inhibitor of RANKL, negatively regulates osteoclasts by inducing apoptosis. ${ }^{48}$ Both factors are produced, in part, by osteoblasts. The RANKL/OPG axis determines the fate and activation of osteoclasts, which perform bone resorption. ${ }^{48}$ Osteoblastic differentiation is under the control of multiple transcription factors and under the direct influence of Wnt signaling. ${ }^{49}$ Chemotaxis of these progenitor cells, proliferation and differentiation of osteoblast precursors, and expression of certain regulatory factors are some of the key events involved in bone formation and mineralization.

\section{Bisphosphonates}

Bisphosphonates preferentially incorporate into sites of active bone formation by binding to hydroxyapatite crystals, i.e., bone mineral. ${ }^{50}$ After binding to bone mineral, bisphosphonates inhibit breakdown and suppress bone reabsorption. Bisphosphonates exert their antiresorptive effects by inducing osteoclast apoptosis, diminishing bone marrow synthesis of osteoclasts, and decreasing osteoclast activity on bony surfaces (Fig. 2) ${ }^{51}$ Bisphosphonates can be further subdivided into nonnitrogen (first generation) and nitrogen-containing (second and third generation) compounds, whereby the presence of nitrogen increases potency by 10 - to 1000 -fold. ${ }^{50}$ First-generation bisphosphonates (etidronate, clodronate, and tiludronate) exert their effects via incorporation into newly synthesized adenosine triphosphate (ATP) compounds after osteoclastic bone mineral uptake. These compounds are rendered into nonhydrolyzable analogs of ATP, leading to intracellular accumulation and eventually apoptosis due to the inhibition of ATP-dependent processes. ${ }^{16}$ Second- and third-generation bisphosphonates (pamidronate, alendronate, risedronate, zoledronate, and olpandronate) inhibit farnesyl pyrophosphate synthase, an important enzyme in the mevalonate pathway; this leads to attenuation of bone resorption and eventually apoptosis..$^{50,52,53}$

\section{Denosumab}

Denosumab is a fully human monoclonal antibody that is analogous to endogenous OPG. OPG suppresses osteoclast proliferation and bone resorption, thus classifying denosumab as an antiresorptive agent (Fig. 2). The mechanism of action involves denosumab binding to RANKL, thus preventing its attachment to RANK. This subsequently leads to RANKL antagonism and inhibits differentiation of early osteoclast precursors into multinucleated osteoclasts, which normally function to induce osteolysis. ${ }^{54}$ RANK/RANKL/OPG system imbalance is characteristic of the pathophysiology of osteoporosis and other metabolic bone diseases. ${ }^{55}$

\section{Romosozumab}

Osteoanabolic agents promote new bone formation, unlike antiresorptive agents, and warrant consideration for individuals with severe osteoporosis. ${ }^{56}$ Romosozumab, a humanized monoclonal antibody, utilizes a different pathway than denosumab and binds to and specifically inhibits the osteocyte-secreted protein, sclerostin. Sclerostin physiologically functions to negatively regulate osteoblasts, and inhibition of this protein thereby promotes bone formation (Fig. 2). ${ }^{57}$ Preclinical studies of sclerostin-deficient rodents resulted in mice with significantly elevated bone mass, increased trabecular and cortical bone formation, and increased overall bone strength. ${ }^{58}$ Romosozumab is the newest of the three osteoanabolic agents currently approved by the US FDA for the treatment of osteoporosis. ${ }^{56}$

\section{Teriparatide}

The osteogenic effect of teriparatide is due to preferential activation of osteoblasts via cell surface receptors for PTH (Fig. 2). This activation induces a myriad of growth factors, including insulin-like growth factor-1, resulting in increased quantities of cancellous bone. ${ }^{59}$ Preclinical testing of teriparatide showed that its administration resulted in enhanced fracture healing, faster rates of bone healing, and new bone formation. ${ }^{16}$ Studies of the use of teriparatide after administration of bisphosphonates have shown that while the action of teriparatide is slightly dampened, it maintains its anabolic properties. ${ }^{60}$ It is important to recognize that the anabolic benefits of teriparatide are quickly lost. Consequently, after its discontinuation, patients with osteoporosis should be prescribed an antiresorptive 

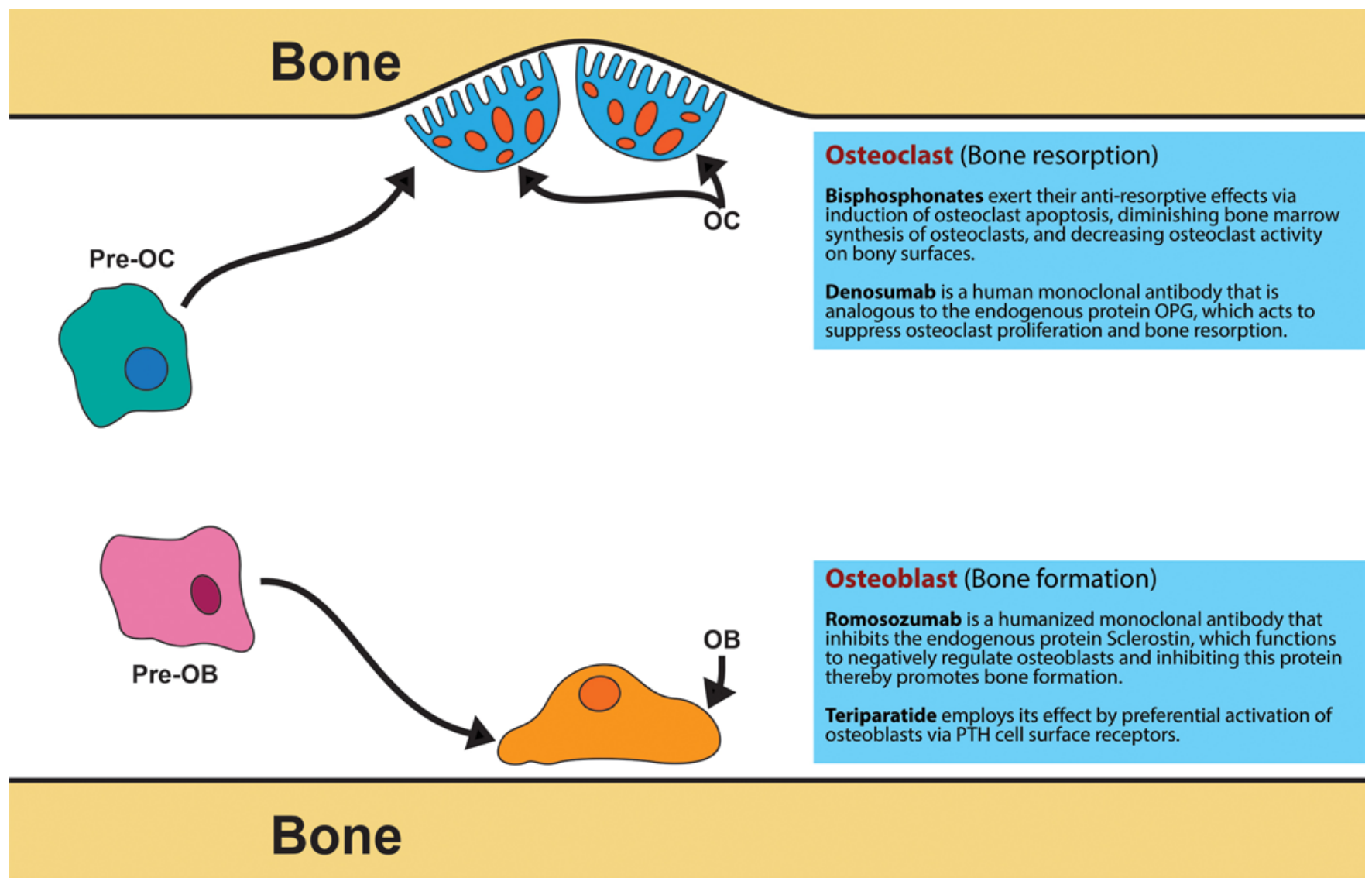

FIG. 2. Illustration depicting the mechanisms of action of antiresorptive and anabolic agents and their effects on osteoclasts or osteoblasts. $\mathrm{OB}=$ osteoblast; $\mathrm{OC}=$ osteoclast; $\mathrm{Pre}-\mathrm{OB}=$ preosteoblast; $\mathrm{Pre}-\mathrm{OC}=$ preosteoclast.

agent. ${ }^{61}$ Teriparatide was the first anabolic agent approved by the US FDA for the treatment of osteoporosis.

\section{Future Directions and Monoclonal Antibodies}

Currently there are two monoclonal antibodies that have been approved by the US FDA for use in humans: denosumab and romosozumab.

Denosumab requires subcutaneous injection (Table 4). ${ }^{54}$ Early studies of the use of denosumab to treat osteoporosis yielded promising results. ${ }^{62-64}$ The landmark 36-month clinical trial by Cummings et al. showed that biannual denosumab administration in postmenopausal women with osteoporosis significantly reduced the risks of vertebral fracture, hip fracture, and nonvertebral fracture. ${ }^{62}$ The 10 year results of the large-scale clinical trial Fracture Reduction Evaluation of Denosumab in Osteoporosis Every 6 Months (FREEDOM) supported these previous conclusions. Investigators found that denosumab was associated with minimal adverse events, decreased incidence of vertebral and nonvertebral fractures, and increased BMD at the lumbar spine by $21.7 \% .^{63}$ Ide et al. studied the effect of teriparatide alone versus teriparatide plus denosumab in 16 patients with osteoporosis who underwent PLIF. ${ }^{65}$ At the 3-, 6-, 9-, and 12-month follow-ups, investigators recorded $\mathrm{BMD}$ at the femoral neck, quantified bone turn- over, and measured biochemical markers, including alkaline phosphatase and type I collagen. Additionally, successful fusion was evaluated radiographically. Although there was no significant difference in biomarkers between the combination treatment and teriparatide groups, radiographic evaluation revealed that the bone union rate $(82 \%$ vs $36 \%, p<0.05)$ and spinal fusion rate $(p<0.05)$ were significantly higher in the combination treatment group at 6 months.

Regarding the adverse effect profile, the FREEDOM trial reported that eczema and flatulence occurred at incidence rates of $2 \%$ or greater, whereas cellulitis and concussion-like symptoms occurred at incidence rates of $0.1 \%$ or greater. ${ }^{46}$ Results from other randomized controlled trials found increased rates of gastrointestinal tract, urinary tract, skin, and ear infections in patients treated with denosumab compared with control patients. ${ }^{55,66}$ None of the phase III trials observed osteonecrosis of the jaw, but it was described in a case report and in an oncological study of patients with metastatic disease who received medical management with denosumab..$^{67,68}$

Given the success of this agent in large-scale randomized controlled trials that enrolled patients with osteoporosis, further evaluation of denosumab as a medical therapy after spine surgery is warranted.

Romosozumab is the newer of the two monoclonal anti- 
bodies that gained US FDA approval in 2019. Its discovery was based on its ability to function as a sclerostin-binding antibody. Preclinical studies of antisclerostin antibodies showed that romosozumab administration resulted in substantial anabolic response of bone formation in rats and monkeys. ${ }^{69}$ The success of romosozumab in the preclinical setting led to further evaluation in randomized clinical trials. The Fracture Study in Postmenopausal Women with Osteoporosis (FRAME) trial, which enrolled over 7000 women with osteoporosis, revealed that those treated with romosozumab demonstrated significantly reduced risk of vertebral fracture compared with control patients. ${ }^{70}$ The Active-Controlled Fracture in Postmenopausal Women with Osteoporosis at High Risk (ARCH) trial examined over 4000 women with osteoporosis who were at higher risk for fracture than the patients in the FRAME study and compared romosozumab with alendronate. ${ }^{71}$ After 12 months of treatment with romosozumab, the experimental group had significantly decreased risk of new vertebral fracture ( $\mathrm{p}<0.001)$, decreased risk of any clinical fracture $(\mathrm{p}<0.001)$, and decreased risk of nonvertebral fracture ( $\mathrm{p}$ $=0.04)$ than the alendronate-treated cohort. ${ }^{72}$

Among the three large-scale randomized controlled trials, injection site reaction was the most commonly cited adverse effect when compared with placebo, followed by serious cardiovascular events. ${ }^{73}$

\section{Conclusions}

Treatment of patients with osteoporosis undergoing spinal surgery is a complex topic that has yet to be fully elucidated. It is imperative for spine surgeons to understand the mechanisms of action of these drugs and their effects on the biology of fusion. Regarding evaluations of efficacy by clinical studies in humans, bisphosphonates and teriparatide appear to be the most frequently studied medications. As data for newer medications become available, the medical management landscape of this unique patient population is likely to expand and result in improved surgical outcomes.

\section{References}

1. Wright NC, Saag KG, Dawson-Hughes B, et al. The impact of the new National Bone Health Alliance (NBHA) diagnostic criteria on the prevalence of osteoporosis in the USA. Osteoporos Int. 2017;28(4):1225-1232.

2. Office of the Surgeon General. Bone Health and Osteoporosis: A Report of the Surgeon General. Office of the Surgeon General; 2004.

3. Siddiqui NA, Shetty KR, Duthie EH Jr. Osteoporosis in older men: discovering when and how to treat it. Geriatrics. 1999; 54(9):20-22, 27-28, 30 passim.

4. Hassanzadeh H, Puvanesarajah V, Dalkin AC. Medical management of osteoporosis for elective spine surgery. Clin Spine Surg. 2016;29(4):134-140.

5. Campion JM, Maricic MJ. Osteoporosis in men. Am Fam Physician. 2003;67(7):1521-1526.

6. Tian L, Yang R, Wei L, et al. Prevalence of osteoporosis and related lifestyle and metabolic factors of postmenopausal women and elderly men: a cross-sectional study in Gansu province, Northwestern of China. Medicine (Baltimore). 2017;96(43):e8294.

7. Kurra S, Fink DA, Siris ES. Osteoporosis-associated fracture and diabetes. Endocrinol Metab Clin North Am. 2014;43(1): 233-243.

8. DeWald CJ, Stanley T. Instrumentation-related complications of multilevel fusions for adult spinal deformity patients over age 65: surgical considerations and treatment options in patients with poor bone quality. Spine (Phila Pa 1976). 2006; 31(19)(suppl):S144-S151.

9. Paxinos O, Tsitsopoulos PP, Zindrick MR, et al. Evaluation of pullout strength and failure mechanism of posterior instrumentation in normal and osteopenic thoracic vertebrae. $J$ Neurosurg Spine. 2010;13(4):469-476.

10. Leichtle CI, Lorenz A, Rothstock S, et al. Pull-out strength of cemented solid versus fenestrated pedicle screws in osteoporotic vertebrae. Bone Joint Res. 2016;5(9):419-426.

11. Okuyama K, Sato K, Abe E, et al. Stability of transpedicle screwing for the osteoporotic spine. An in vitro study of the mechanical stability. Spine (Phila Pa 1976). 1993;18(15): $2240-2245$.

12. Halvorson TL, Kelley LA, Thomas KA, et al. Effects of bone mineral density on pedicle screw fixation. Spine (Phila Pa 1976). 1994;19(21):2415-2420.

13. Soshi S, Shiba R, Kondo H, Murota K. An experimental study on transpedicular screw fixation in relation to osteoporosis of the lumbar spine. Spine (Phila Pa 1976). 1991;16(11): $1335-1341$.

14. Cho JH, Hwang CJ, Kim H, et al. Effect of osteoporosis on the clinical and radiological outcomes following one-level posterior lumbar interbody fusion. J Orthop Sci. 2018;23(6): 870-877.

15. Yagi M, King AB, Boachie-Adjei O. Incidence, risk factors, and natural course of proximal junctional kyphosis: surgical outcomes review of adult idiopathic scoliosis. Minimum 5 years of follow-up. Spine (Phila Pa 1976). 2012;37(17): $1479-1489$.

16. Chaudhary N, Lee JS, Wu JY, Tharin S. Evidence for use of teriparatide in spinal fusion surgery in osteoporotic patients. World Neurosurg. 2017;100:551-556.

17. Ohtori S, Inoue G, Orita S, et al. Comparison of teriparatide and bisphosphonate treatment to reduce pedicle screw loosening after lumbar spinal fusion surgery in postmenopausal women with osteoporosis from a bone quality perspective. Spine (Phila Pa 1976). 2013;38(8):E487-E492.

18. Inoue $G$, Ueno M, Nakazawa $T$, et al. Teriparatide increases the insertional torque of pedicle screws during fusion surgery in patients with postmenopausal osteoporosis. J Neurosurg Spine. 2014;21(3):425-431.

19. Ohtori S, Orita S, Yamauchi K, et al. More than 6 months of teriparatide treatment was more effective for bone union than shorter treatment following lumbar posterolateral fusion surgery. Asian Spine J. 2015;9(4):573-580.

20. Tsai JN, Uihlein AV, Lee H, et al. Teriparatide and denosumab, alone or combined, in women with postmenopausal osteoporosis: the DATA study randomised trial. Lancet. 2013;382(9886):50-56.

21. Park SB, Kim CH, Hong M, et al. Effect of a selective estrogen receptor modulator on bone formation in osteoporotic spine fusion using an ovariectomized rat model. Spine $J$. 2016;16(1):72-81.

22. Yasen M, Li X, Jiang L, et al. Effect of zoledronic acid on spinal fusion outcomes in an ovariectomized rat model of osteoporosis. J Orthop Res. 2015;33(9):1297-1304.

23. Nakao S, Minamide A, Kawakami M, et al. The influence of alendronate on spine fusion in an osteoporotic animal model. Spine (Phila Pa 1976). 2011;36(18):1446-1452.

24. Yishake M, Yasen M, Jiang L, et al. Effects of combined teriparatide and zoledronic acid on posterior lumbar vertebral fusion in an aged ovariectomized rat model of osteopenia. $J$ Orthop Res. 2018;36(3):937-944.

25. Nagahama K, Kanayama M, Togawa D, et al. Does alendro- 
nate disturb the healing process of posterior lumbar interbody fusion? A prospective randomized trial. J Neurosurg Spine. 2011;14(4):500-507.

26. Park YS, Kim HS, Baek SW, et al. The effect of zoledronic acid on the volume of the fusion-mass in lumbar spinal fusion. Clin Orthop Surg. 2013;5(4):292-297.

27. Tu CW, Huang KF, Hsu HT, et al. Zoledronic acid infusion for lumbar interbody fusion in osteoporosis. J Surg Res. 2014; 192(1):112-116.

28. Chen F, Dai Z, Kang Y, et al. Effects of zoledronic acid on bone fusion in osteoporotic patients after lumbar fusion. Osteoporos Int. 2016;27(4):1469-1476.

29. Seki S, Hirano N, Kawaguchi Y, et al. Teriparatide versus low-dose bisphosphonates before and after surgery for adult spinal deformity in female Japanese patients with osteoporosis. Eur Spine J. 2017;26(8):2121-2127.

30. Kawabata A, Yoshii T, Hirai T, et al. Effect of bisphosphonates or teriparatide on mechanical complications after posterior instrumented fusion for osteoporotic vertebral fracture: a multi-center retrospective study. BMC Musculoskelet Disord. 2020;21(1):420.

31. Oba H, Takahashi J, Yokomichi H, et al. Weekly teriparatide versus bisphosphonate for bone union during 6 months after multi-level lumbar interbody fusion for osteoporotic patients: a multicenter, prospective, randomized study. Spine (Phila Pa 1976). 2020;45(13):863-871.

32. Ding Q, Chen J, Fan J, et al. Effect of zoledronic acid on lumbar spinal fusion in osteoporotic patients. Eur Spine J. 2017; 26(11):2969-2977.

33. Kang T, Park SY, Hong SH, et al. Bone union after spinal fusion surgery using local bone in long-term bisphosphonate users: a prospective comparative study. Arch Osteoporos. 2019;14(1):74.

34. Ohtori S, Inoue G, Orita S, et al. Teriparatide accelerates lumbar posterolateral fusion in women with postmenopausal osteoporosis: prospective study. Spine (Phila Pa 1976). 2012; 37(23):E1464-E1468.

35. Reyes C, Hitz M, Prieto-Alhambra D, Abrahamsen B. Risks and benefits of bisphosphonate therapies. J Cell Biochem. 2016;117(1):20-28.

36. Han SL, Wan SL. Effect of teriparatide on bone mineral density and fracture in postmenopausal osteoporosis: metaanalysis of randomised controlled trials. Int J Clin Pract. 2012;66(2):199-209.

37. Ebata S, Takahashi J, Hasegawa T, et al. Role of weekly teriparatide administration in osseous union enhancement within six months after posterior or transforaminal lumbar interbody fusion for osteoporosis-associated lumbar degenerative disorders: a multicenter, prospective randomized study. $J$ Bone Joint Surg Am. 2017;99(5):365-372.

38. Cho PG, Ji GY, Shin DA, et al. An effect comparison of teriparatide and bisphosphonate on posterior lumbar interbody fusion in patients with osteoporosis: a prospective cohort study and preliminary data. Eur Spine J. 2017;26(3):691-697.

39. Kim JW, Park SW, Kim YB, Ko MJ. The effect of postoperative use of teriparatide reducing screw loosening in osteoporotic patients. J Korean Neurosurg Soc. 2018;61(4):494-502.

40. Sugiura T, Kashii M, Matsuo Y, et al. Intermittent administration of teriparatide enhances graft bone healing and accelerates spinal fusion in rats with glucocorticoid-induced osteoporosis. Spine J. 2015;15(2):298-306.

41. Lawrence JP, Ennis F, White AP, et al. Effect of daily parathyroid hormone (1-34) on lumbar fusion in a rat model. Spine J. 2006;6(4):385-390.

42. Qiu Z, Wei L, Liu J, et al. Effect of intermittent PTH (1-34) on posterolateral spinal fusion with iliac crest bone graft in an ovariectomized rat model. Osteoporos Int. 2013;24(10): 2693-2700.

43. O'Loughlin PF, Cunningham ME, Bukata SV, et al. Para- thyroid hormone (1-34) augments spinal fusion, fusion mass volume, and fusion mass quality in a rabbit spinal fusion model. Spine (Phila Pa 1976). 2009;34(2):121-130.

44. Lehman RA Jr, Dmitriev AE, Cardoso MJ, et al. Effect of teriparatide [rhPTH $(1,34)]$ and calcitonin on intertransverse process fusion in a rabbit model. Spine (Phila Pa 1976). 2010; 35(2):146-152.

45. Black DM, Rosen CJ. Clinical practice. Postmenopausal osteoporosis. N Engl J Med. 2016;374(3):254-262.

46. Rizzoli R, Reginster JY, Boonen S, et al. Adverse reactions and drug-drug interactions in the management of women with postmenopausal osteoporosis. Calcif Tissue Int. 2011; 89(2):91-104.

47. Boden SD. Overview of the biology of lumbar spine fusion and principles for selecting a bone graft substitute. Spine (Phila Pa 1976). 2002;27(16)(suppl 1):S26-S31.

48. Theoleyre S, Wittrant Y, Tat SK, et al. The molecular triad OPG/RANK/RANKL: involvement in the orchestration of pathophysiological bone remodeling. Cytokine Growth Factor Rev. 2004;15(6):457-475.

49. Baron R, Rawadi G, Roman-Roman S. Wnt signaling: a key regulator of bone mass. Curr Top Dev Biol. 2006;76:103-127.

50. Drake MT, Clarke BL, Khosla S. Bisphosphonates: mechanism of action and role in clinical practice. Mayo Clin Proc. 2008;83(9):1032-1045.

51. Rodan GA, Fleisch HA. Bisphosphonates: mechanisms of action. J Clin Invest. 1996;97(12):2692-2696.

52. Zameer S, Najmi AK, Vohora D, Akhtar M. Bisphosphonates: future perspective for neurological disorders. Pharmacol Rep. 2018;70(5):900-907.

53. Rodriguez JB, Falcone BN, Szajnman SH. Approaches for designing new potent inhibitors of farnesyl pyrophosphate synthase. Expert Opin Drug Discov. 2016;11(3):307-320.

54. Baron R, Ferrari S, Russell RGG. Denosumab and bisphosphonates: different mechanisms of action and effects. Bone. 2011;48(4):677-692.

55. Anastasilakis AD, Toulis KA, Goulis DG, et al. Efficacy and safety of denosumab in postmenopausal women with osteopenia or osteoporosis: a systematic review and a metaanalysis. Horm Metab Res. 2009;41(10):721-729.

56. Haas AV, LeBoff MS. Osteoanabolic agents for osteoporosis. J Endocr Soc. 2018;2(8):922-932.

57. Padhi D, Jang G, Stouch B, et al. Single-dose, placebo-controlled, randomized study of AMG 785, a sclerostin monoclonal antibody. J Bone Miner Res. 2011;26(1):19-26.

58. Li X, Ominsky MS, Niu QT, et al. Targeted deletion of the sclerostin gene in mice results in increased bone formation and bone strength. J Bone Miner Res. 2008;23(6):860-869.

59. Hirsch BP, Unnanuntana A, Cunningham ME, Lane JM. The effect of therapies for osteoporosis on spine fusion: a systematic review. Spine J. 2013;13(2):190-199.

60. Cosman F. Anabolic and antiresorptive therapy for osteoporosis: combination and sequential approaches. Curr Osteoporos Rep. 2014;12(4):385-395.

61. Black DM, Bilezikian JP, Ensrud KE, et al. One year of alendronate after one year of parathyroid hormone (1-84) for osteoporosis. N Engl J Med. 2005;353(6):555-565.

62. Cummings SR, San Martin J, McClung MR, et al. Denosumab for prevention of fractures in postmenopausal women with osteoporosis. N Engl J Med. 2009;361(8):756-765.

63. Bone HG, Wagman RB, Brandi ML, et al. 10 years of denosumab treatment in postmenopausal women with osteoporosis: results from the phase 3 randomised FREEDOM trial and open-label extension. Lancet Diabetes Endocrinol. 2017; 5(7):513-523.

64. Chitre M, Shechter D, Grauer A. Denosumab for treatment of postmenopausal osteoporosis. Am J Health Syst Pharm. 2011; 68(15):1409-1418.

65. Ide M, Yamada K, Kaneko K, et al. Combined teriparatide 
and denosumab therapy accelerates spinal fusion following posterior lumbar interbody fusion. Orthop Traumatol Surg Res. 2018;104(7):1043-1048.

66. Toulis KA, Anastasilakis AD. Increased risk of serious infections in women with osteopenia or osteoporosis treated with denosumab. Osteoporos Int. 2010;21(11):1963-1964.

67. Taylor KH, Middlefell LS, Mizen KD. Osteonecrosis of the jaws induced by anti-RANK ligand therapy. Br J Oral Maxillofac Surg. 2010;48(3):221-223.

68. Stopeck AT, Lipton A, Body JJ, et al. Denosumab compared with zoledronic acid for the treatment of bone metastases in patients with advanced breast cancer: a randomized, doubleblind study. J Clin Oncol. 2010;28(35):5132-5139.

69. Ominsky MS, Boyce RW, Li X, Ke HZ. Effects of sclerostin antibodies in animal models of osteoporosis. Bone. 2017;96: 63-75.

70. Cosman F, Crittenden DB, Adachi JD, et al. Romosozumab treatment in postmenopausal women with osteoporosis. $N$ Engl J Med. 2016;375(16):1532-1543.

71. Möckel L, Bartneck M, Möckel C. Risk of falls in postmenopausal women treated with romosozumab: Preliminary indices from a meta-analysis of randomized, controlled trials. Osteoporos Sarcopenia. 2020;6(1):20-26.

72. Saag KG, Petersen J, Brandi ML, et al. Romosozumab or alendronate for fracture prevention in women with osteoporosis. N Engl J Med. 2017;377(15):1417-1427.

73. Kerschan-Schindl K. Romosozumab: a novel bone anabolic treatment option for osteoporosis? Wien Med Wochenschr. 2020;170(5-6):124-131.

\section{Disclosures}

Dr. Wang receives royalty payments from DePuy-Synthes Spine, Children's Hospital of Los Angeles, Springer Publishing, and Quality Medical Publishing; is a consultant for DePuy-Synthes Spine, Stryker Spine, K2M, Medtronic, and Spineology; is an advisory board member for Vallum; owns stock in Spinicity, Innovative Surgical Devices, Kinesiometrics, and Medical Device Partners; holds a patent with DePuy-Synthes; and receives grants from the US Department of Defense.

\section{Author Contributions}

Conception and design: Perez-Roman, Burks, Wang. Acquisition of data: Bryant, Perez-Roman, Wang. Analysis and interpretation of data: Perez-Roman, Burks. Drafting the article: Bryant, PerezRoman. Critically revising the article: Burks, Wang. Reviewed submitted version of manuscript: Bryant, Wang.

\section{Correspondence}

Jean-Paul Bryant: Lois Pope Life Center, Miami, FL. jxb1400@ med.miami.edu. 